\title{
Lower production of IL-17A and increased susceptibility to Mycobacterium bovis in mice coinfected with Strongyloides venezuelensis
}

\author{
Alyria Teixeira Diass ${ }^{1}$, Sandra Bertelli Ribeiro de Castro', Caio César Souza Alves ${ }^{1}$, \\ Alice Belleigoli Rezende', Michele Fernandes Rodrigues', Rachel Rocha Pinheiro Machado', \\ Adriana Fernandes ${ }^{2}$, Deborah Negrão-Corrêa ${ }^{2}$, Henrique Couto Teixeiraa ${ }^{1}$, Ana Paula Ferreira ${ }^{1 /+}$ \\ 'Departamento de Parasitologia, Microbiologia e Imunologia, Instituto de Ciências Biológicas, Universidade Federal de Juiz de Fora, \\ 36036-330 Juiz de Fora, MG, Brasil ²Departamento de Parasitologia, Instituto de Ciências Biológicas, \\ Universidade Federal de Minas Gerais, Belo Horizonte, MG, Brasil
}

The presence of intestinal helminths can down-regulate the immune response required to control mycobacterial infection. BALB/c mice infected with Mycobacterium bovis following an infection with the intestinal helminth Strongyloides venezuelensis showed reduced interleukin-17A production by lung cells and increased bacterial burden. Also, small granulomas and a high accumulation of cells expressing the inhibitory molecule CTLA-4 were observed in the lung. These data suggest that intestinal helminth infection could have a detrimental effect on the control of tuberculosis $(T B)$ and render coinfected individuals more susceptible to the development of TB.

Key words: M. bovis - S. venezuelensis - coinfection - IL-17 - CTLA-4 - tuberculosis

One-third of the world's population is infected with Mycobacterium tuberculosis (MTB) and 8.9-9.9 million new cases of tuberculosis (TB) were reported in 2008 (WHO 2009). Whereas MTB is the main human pathogen, Mycobacterium bovis has a broad range of hosts and remains a serious zoonotic threat to human health (Probst et al. 2011).

Interleukin-17 (IL-17), mainly produced by a subset of pro-inflammatory $\mathrm{CD} 4^{+} \mathrm{T}$ cells known as Th17 cells, has been associated with protection against TB. It has been suggested that they make an important contribution to the human antimycobacterial immune response (Khader et al. 2007, Khader \& Cooper 2008, Scriba et al. 2008). IL-17A contributes to the immune response against mycobacterial infection, especially the infection-induced granuloma formation (Umemura et al. 2007, Yoshida et al. 2010).

The protective efficacy of BCG vaccination against virulent MTB is significantly impaired in helminth-infected animals (Elias et al. 2005). It has been shown that adolescents with helminth infections who took the second dose of BCG had decreased BCG-induced interferon-gamma (IFN- $\gamma$ ) response, indicating that worm infections may impair both the Th1 response against TB and the efficacy of BCG vaccination (Ferreira et al. 2002). However, the impact of worm-induced immunomodulation on immunity against TB and on the efficacy of BCG vaccination remains to be clarified.

Financial support: CNPq, FAPEMIG, CAPES

+Corresponding author: ana.paula@ufjf.edu.br

Received 10 December 2010

Accepted 6 June 2011
Strongyloides venezuelensis, a murine intestinal parasite, has been used as a model for understanding the host-parasite relationship in strongyloidiasis (Fernandes et al. 2008). The aim of this study was to evaluate whether $S$. venezuelensis coinfection can modulate $M$. bovis infection, affecting IL-17A production and granuloma formation in $\mathrm{BALB} / \mathrm{c}$ mice.

BALB/c mice were subcutaneously inoculated with 700 third-stage larvae of $S$. venezuelensis $/ 0.1 \mathrm{~mL}$ phosphate buffered saline in the abdominal region. Three days after $S$. venezuelensis inoculation, mice were intravenously (i.v.) inoculated with $1 \times 10^{5}$ colony forming units (CFU) of M. bovis (ATCC 19274). The mice were divided into four groups based on the inoculum used: (i) S. venezuelensis (Sv) alone, (ii) M. bovis (Mb) alone, (iii) $\mathrm{Sv}$ and $\mathrm{Mb}$ coinfection (CI) and (iv) an uninfected control group. All procedures were performed in accordance with the principles of the Brazilian Code for the Use of Laboratory Animals. This project was approved by the Ethical Committee of Federal University of Juiz de Fora on the use of laboratory animals (034/2006).

Twenty-eight days after $M$. bovis infection, mice were sacrificed and their lungs were removed and homogenised in distilled water. Ten-fold serial dilutions of the homogenates were placed onto Lowenstein-Jensen agar (Difco, Sparks, MD, USA). After 21 days of incubation at $37^{\circ} \mathrm{C}$ and $5 \% \mathrm{CO}_{2}$, the colonies were counted and bacterial counts in the organs were calculated as $\log _{10} \mathrm{CFU}$ per organ.

IL-17A concentrations in lung homogenates were determined 28 days after $M$. bovis infection by enzyme linked immunosorbent assay, according to the manufacturer's instructions (eBioscience, San Diego, CA, USA). Lung cells were incubated with anti-CD3-PerCP and anti-CTLA-4-PE mAb (BD Biosciences Pharmingen). Staining was analysed using a FACSCalibur flow cytometer and CellQuest software (Becton Dickinson, San 
Jose, CA, USA). Hamster IgG1 was used as the isotype control for staining (BD Biosciences Pharmingen). For histological evaluation, lungs were fixed in $10 \%$ formaldehyde before processing and then embedded in paraffin. From each block, 5 - $\mu$ m-thick histological sections were cut and stained with haematoxylin-eosin.

Data are reported as mean value \pm standard error and were analysed using Mann-Whitney test (GraphPad Prism 5.00). The differences were considered significant at $p<0.05$. Results are representative of two independent experiments.

At day seven post-inoculation, $S$. venezuelensis infection was monitored by counting the number of eggs per gram of faeces in both Sv and CI groups. The number of eggs was higher in the CI group (65.4 \pm 11.51$)$ in comparison to the $\mathrm{Sv}$ group $(37.5 \pm 6.72)$. The results are statistically significant $(\mathrm{p}<0.05)$ and are consistent with previously reported results by Carmo et al. (2009).

To assess the level of immune protection against pulmonary mycobacterial infection, the viable bacteria count and histological examination of the lungs was determined 28 days after challenge with $M$. bovis. The bacterial load was $4.961 \mathrm{log}$ in the CI group and 3.618
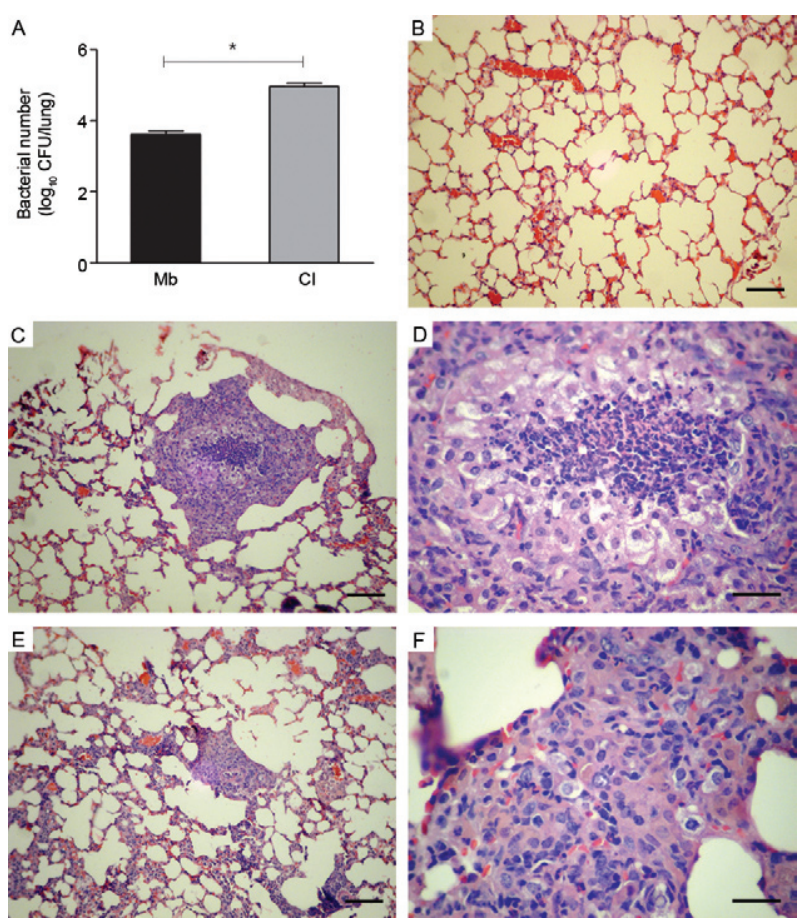

Fig. 1: number of Mycobacterium bovis-colony forming units (CFU) and photomicrograph of lung stained with haematoxylin-eosin. Three days after subcutaneously infection with 700 L3 of Strongyloides venezuelensis, $\mathrm{BALB} / \mathrm{c}$ mice were intravenously coinfected with $10^{5}$ M. bovis [S. venezuelensis and M. bovis (Mb) coinfection (CI) group]. At day 28 post- $M$. bovis infection, the number of CFUs per lung was determined (A). B: photomicrograph of lung tissue of non-infected mice; C, D: M. bovis-infected mice; E, F: coinfected mice. Bar in A represents the mean value \pm standard error of five mice per group and results are representative of two independent experiments. Asterisk indicates statistically significant differences ( $p<0.05$, Mann-Whitney test). Bar $=60 \mu \mathrm{m}(\mathrm{B}, \mathrm{C}, \mathrm{E})$ and $20 \mu \mathrm{m}(\mathrm{D}, \mathrm{F})$. $\log$ in the $\mathrm{Mb}$ group. The $\mathrm{CI}$ group showed significantly higher $\mathrm{CFU}$ compared to the $\mathrm{Mb}$ infected group ( $\mathrm{p}<$ 0.05) (Fig. 1A).

Examination of lung histological sections from the $\mathrm{Mb}$ infected group reveals the presence of mature granulomas at the peribronchiolar site, with a polymorphonuclear-rich centre and surrounded by macrophages with vacuolated cytoplasm (Fig. 1B-D). The lungs from mice in the CI group showed diffuse infiltration of mononuclear cells and small granulomas in the parenchyma (Fig. 1E, F).

The impaired IL-17A production and number of lung cells expressing CTLA-4 were measured 28 days after i.v. infection with $M$. bovis. IL-17A production in the CI group was significantly less than in the $\mathrm{Mb}$ group $(\mathrm{p}<$ $0.05)$ (Fig. 2A). Levels of IFN- $\gamma$ and tumor necrosis factor-alpha did not differ between the $\mathrm{CI}$ and $\mathrm{Mb}$ groups (Supplementary data). Numbers of $\mathrm{CD}^{+}$CTLA- $4^{+}$cells in the lung were higher in the CI group in comparison to the $\mathrm{Mb}$ group $(\mathrm{p}<0.05)$ (Fig. $2 \mathrm{~B})$.

In the present study, concomitant infection with $M$. bovis and the intestinal helminth $S$. venezuelensis in $\mathrm{BALB} / \mathrm{c}$ mice was associated with decreased IL-17A production in lungs. In addition, coinfected mice showed increased bacterial burden and an increased absolute number of cells expressing CTLA-4 in the lungs. These results suggest a critical role of IL-17A in controlling $M$. bovis infection, which appears to be modulated by concomitant helminth infection.

The reduction of the IL-17A levels and the presence of only small granulomas observed in coinfected mice could be a factor involved in the increased susceptibility to $M$. bovis infection in this model. IL-17, the main cytokine produced by Th17 cells (Zhou et al. 2007), has an important role in initiating inflammation, recruiting neutrophils and inducing the formation of granulomas at the site of MTB infection (Stark et al. 2005, Lockhart et al. 2006). The reduction of neutrophils in mycobacterial infection has been associated with increased bacterial burden in the lung (Fulton et al. 2002).

Gamma-delta T cells have been identified as the major IL-17 producer in mycobacterial infection (Lockhart et al. 2006, Umemura et al. 2007). These cells play an impor-
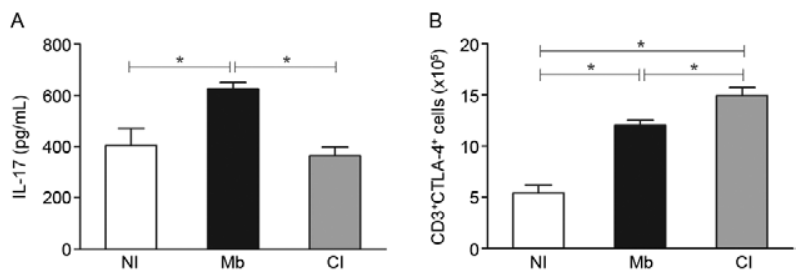

Fig. 2: detection of interleukin (IL)-17 in lungs of non-infected mice (NI), only Mycobacterium bovis-infected mice (Mb) and in Strongyloides venezuelensis plus $M$. bovis coinfected mice (CI). Twenty eight days after intravenously $M$. bovis infection, the production of IL-17A (A) and the absolute number of cells expressing CTLA-4 (B) was determined. Each bar represents the mean value \pm standard error of five mice per group and results are representative of two independent experiments. Asterisks indicate statistically significant differences $(p<$ 0.05, Mann-Whitney test). 
tant role in the maturation of granulomas in BCG-infected lungs and the adoptive transfer of these cells successfully reconstitutes the capacity of IL-17A knockout mice (IL$17^{-/}$) to develop mature granulomas (Yoshida et al. 2010). Furthermore, the size and number of granulomas in the lungs of IL-17/- mice were reduced in comparison to the granulomas in wild-type mice on day 28 of infection. This resulted in a reduction of IFN- $\gamma$ production by $\mathrm{CD}^{+}$ $\mathrm{T}$ cells, causing impaired granuloma formation in $M$. bovis $\mathrm{BCG}$ infection (Umemura et al. 2007, Yoshida et al. 2010). Additionally, the lung granulomas of IL-17-/ mice were less densely packed with mononuclear cells than those in the wild-type mice (Umemura et al. 2007).

In the present study, an increase in the absolute number of cells expressing CTLA-4 was observed in lungs of mice with concomitant infection. CTLA-4 is expressed by all $\mathrm{T}$ lymphocytes and is able to bind to CD80/CD86 in antigen-presenting cells, competing with the coactivation molecule CD28 (Saverino et al. 1999). In mycobacterial infection, the role of CTLA-4 has been investigated. Blocking CTLA-4 increases the protective immune response against $\mathrm{TB}$, whereas increasing CTLA-4 expression promotes susceptibility to infection (Kirman et al. 1999). In a recent study, the presence of patent filarial infection in individuals with coexisting latent MTB infection altered the M. tuberculosis-specific immune responses, diminished the production of IL-17 and increased CTLA-4 expression. Furthermore, CTLA-4 blockade significantly increased the production of both Th1 and Th17 cytokines (Babu et al. 2009).

In conclusion, the increased bacterial burden in the lung of CI mice observed in this study could be related to reduced IL-17A production and to the increased absolute number of cells that expressed CTLA-4. This is the first study that shows the importance of IL-17A in experimental models of concomitant infections. Further investigation would be worthwhile into pathways involving IL-17A and CTLA-4 that modulate the immune response to helminth infections, which can alter susceptibility to mycobacterial infections.

\section{REFERENCES}

Babu S, Bhat SQ, Kumar NP, Jayantarsi S, Rukmani S, Kumaran P, Gopi PGP, Kolappan C, Kumaraswami V, Nutman TB 2009. Human type 1 and 17 responses in latent tuberculosis are modulated by coincident filarial infection through cytotoxic T lymphocyte antigen-4 and programmed death-1. J Infect Dis 200: 288-298.

Carmo AM, Vicentini MA, Dias AT, Alves LL, Alves CCS, Brandi JS, De Paula ML, Fernandes A, Barsante MM, Souza MA, Teixeira HC, Negrão-Corrêa D, Ferreira AP 2009. Increased susceptibility to Strongyloides venezuelensis in mice due to Mycobacterium bovis co-infection which modulates production of Th2 cytokines. Parasitology 136: 1357-365.

Elias D, Akuffo H, Pawlowski A, Haile M, Schon T, Britton S 2005. Schistosoma mansoni infection reduces the protective efficacy of BCG vaccination against virulent Mycobacterium tuberculosis. Vaccine 23: 1326-334.
Fernandes A, Ferreira ATM, Eschenazi PD, Schilter HC, Sousa ALS, Teixeira MM, Negrão-Corrêa D 2008. Evaluation of the immune response against Strongyloides venezuelensis in antigen-immunized or previously infected mice. Parasite Immunol 30: 139-149.

Ferreira AP, Aguiar AS, Fava MWB, Corrêa JOA, Teixeira FM, Teixeira HC 2002. Can the efficacy of Bacille Calmette-Guérin tuberculosis vaccine be affected by intestinal parasitic infections? J Infect Dis 186: 441-442.

Fulton SA, Reba SM, Martin TD, Boom WH 2002. Neutrophil-mediated mycobacteriocidal immunity in the lung during Mycobacterium bovis BCG infection in C57BL/6 mice. Infect Immun 70: 5322-5327.

Khader S, Bell G, Pearl J, Fountain J, Rangel-Moreno J, Cilley G, Shen F, Eaton S, Gaffen S, Swain S, Locksley R, Haynes L, Randall T, Cooper A 2007. IL-23 and IL-17 in establishment of protective pulmonary $\mathrm{CD}^{+} \mathrm{T}$ cell responses upon vaccination and during Mycobacterium tuberculosis challenge. Nat Immunol 8: 369-377.

Khader SA, Cooper AM 2008. IL-23 and IL-17 in tuberculosis. $C y$ tokine 41: 79-83.

Kirman J, McCoy K, Hook S, Prout M, Delahunt B, Orme I, Frank A, Le Gros G 1999. CTLA-4 blockade enhances the immune response induced by mycobacterial infection, but does not lead to increased protection. Infect Immun 67: 3786-3792.

Lockhart E, Green AM, Flyn JL 2006. IL-17 production is dominated by \{gamma\} \{delta\} T cells rather than CD4 T cells during Mycobacterium tuberculosis infection. J Immunol 177: 4662-4669.

Probst C, Freuling C, Moser I, Geue L, Köhler H, Conraths FJ, Hotzel H, Liebler-Tenorio EM, Kramer M 2011. Bovine tuberculosis: making a case for effective surveillance. Epidemiol Infect 139: 105-112.

Saverino D, Tenca C, Zarcone D, Merlo A, Megiovanni AM, Valle MT, Manca F, Grossi CE, Ciccone E 1999. CTLA-4 (CD152) inhibits the specific lysis mediated by human cytolytic T lymphocytes in a clonally distributed fashion. $J$ Immunol 162: 651-658.

Scriba TJ, Kalsdorf B, Abrahams DA, Isaacs F, Hofmeister J, Black G, Hassan HY, Wilkinson RJ, Walzl G, Gelderbloem SJ, Mahomed H, Hussey GD, Hanekom WA 2008. Distinct, specific IL-17- and IL-22-producing $\mathrm{CD}^{+} \mathrm{T}$ cell subsets contribute to the human anti-mycobacterial immune response. J Immunol 180: 1962-1970.

Stark MA, Huo Y, Burcin TL, Morris MA, Olson TS, Ley K 2005. Phagocytosis of apoptotic neutrophils regulates granulopoiesis via IL-23 and IL-17. Immunity 22: 285-294.

Umemura M, Yahagi A, Hamada S, Begum D, Watanabe $\mathrm{H}$, Kawakami K, Suda T, Sudo K, Nakae S, Iwakura Y, Matsuzaki G 2007. IL-17-mediated regulation of innate and acquired immune response against pulmonary Mycobacterium bovis Bacille Calmette-Guérin infection. J Immunol 178: 3786-3796.

WHO - World Health Organization 2009. Global tuberculosis control 2009: surveillance, planning, financing, WHO, Geneva, 303 pp.

Yoshida YO, Umemura M, Yahagi A, O’Brien RL, Ikuta K, Kishihara K, Hara H, Nakae S, Iwakura Y, Matsuzaki G 2010. Essential role of IL-17A in the formation of a mycobacterial infection-induced granuloma in the lung. J Immunol 184: 4414-4422.

Zhou L, Ivanov II, Spolski R, Min R, Shenderov K, Egawa T, Levy DE, Leonard WJ, Littman DR 2007. IL-6 programs T(H)-17 cell differentiation by promoting sequential engagement of the IL-21 and IL-23 pathways. Nat Immunol 8: 967-974. 

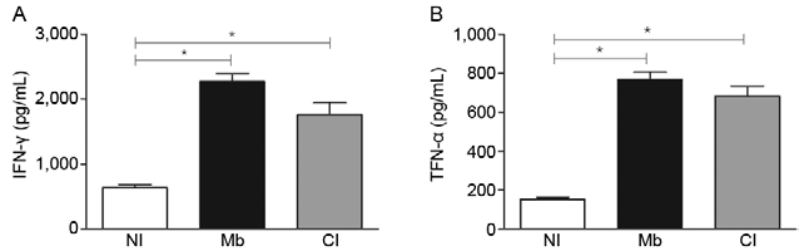

Production of cytokines in lungs of non-infected mice (NI), only Mycobacterium bovis-infected mice (Mb) and in Strongyloides venezuelensis plus M. bovis coinfected mice (CI). Twenty eight days after intravenously M. bovis infection, the production of interferon-gamma (IFN- $\gamma$ ) (A) and tumor necrosis factor-alpha (TNF- $\alpha$ ) (B) was determined. Each bar represents the mean value \pm standard error of five mice per group and results are representative of two independent experiments. Asterisks indicate statistically significant differences ( $p$ $<0.05$, Mann-Whitney test). 